\title{
An anomalous muscular bundle connecting biceps femoris to semitendinosus
}

\begin{abstract}
Anatomical variations of the hamstring muscles are not common. During anatomy dissection of the lower extremities of an adult male cadaver, an aberrant muscular bundle was observed. This aberrant muscle bundle was originated from long head of biceps femoris and merged with semitendinosus. Because the muscular bundle runs across the sciatic nerve, it may compress the sciatic nerve and cause symptoms of sciatic nerve compression. This type of muscular bundle may potentially predispose hamstring strain and hamstring injury. Such a muscular bundle also may cause confusion during surgery or evaluation of CT and MRI scans. Therefore, awareness of this type of variations is essential to avoid complications during diagnostic radiology and surgeries.
\end{abstract}

Keywords: biceps femoris, hamstring muscles, muscle bundle, muscular slip, semitendinosus
Volume 4 Issue 3 - 2017

\author{
Murat Cetkin,' Mustafa Deniz Yoruk, ${ }^{2}$ Murat \\ Golpinar, ${ }^{3}$ Merve Dinc, ${ }^{4}$ Yelda Pinar ${ }^{2}$ \\ 'Department of Anatomy, Gaziantep University, Turkey \\ 2Department of Anatomy, Ege University, Turkey \\ ${ }^{3}$ Department of Anatomy, Ondokuz Mayis University, Turkey \\ ${ }^{4}$ Department of Anatomy, Dumlupinar University, Turkey
} Correspondence: Yelda Pinar, Department of Anatomy, Faculty
of Medicine, Ege University, Izmir, Turkey,

Email yeldapinar@gmail.com

Received: August 23, 2017| Published: October II, 2017

\section{Introduction}

The hamstring muscle group consists of three muscles in the posterior thigh; the semitendinosus (ST) and the semimembranosus (SM) are located at the medial side, the biceps femoris at the lateral side (BF). ${ }^{1}$ The biceps femoris has two heads, which are anatomically and functionally distinct; the short (BFsh) and the long (BFlh) head. The semitendinosus and long head of the biceps femoris have a common origin from medial facet of ischial tuberosity, whereas the semimembranosus has origin from lateral facet of ischial tuberosity separately. ${ }^{2}$ Short head of biceps femoris originates from the linera aspera to join the long head of biceps femoris. The semimembranosus muscle attaches to posteromedial aspect of the tibia while the semitendinosus muscle attach to anteromedial aspect of the tibia by way of a common tendon with gracilis and sartorius muscles. Biceps femoris muscle inserts laterally on the head of the fibula. With the exception of the BFSH, hamstrings are innervated by the tibial part of sciatic nerve. The sciatic nerve passes the proximal hamstring muscle complex on the lateral side at a distance of approximately $1 \mathrm{~cm}$ from most lateral aspect. ${ }^{3}$

The semitendinosus, the semimembranosus, and long head of the biceps femoris cross hip joint proximally and knee joint distally and due to this configuration they are primarily responsible for flexion of the knee joint and as well as assisting the extension of the hip joint. The short head of biceps femoris muscle only crosses the knee joint and, therefore, involved in only knee flexion.

The hamstring muscles act as both hip extensors and knee flexors, making a complex contribution to the gait circle during walking and running which involves absorbing kinetic energy and protecting knee and hip joints. These muscles also contribute knee and hip internal and external rotation, respectively. These muscles are long, biarticular, and involved in many types of locomotion. Anatomical and functional features of Hamstring muscle complex predispose it to injury. ${ }^{3}$ Therefore it is not suprising that injuries of the hamstring muscle complex are more common in many sports and have increased in recent times. ${ }^{4}$

Anatomical variations of the hamstring muscles are not common. The variations in anatomical structures may predispose particular patients to injury that may cause decrease in the normal glide and flexibility of the muscles. ${ }^{5}$

Detail knowledge on the variant anatomy of this region as well as normal anatomy of posterior thigh is necessary for avoid diagnostic errors for surgeons, radiologists or physiotherapists. Therefore, we have reported here anomalous muscular bundle which connecting long head biceps femoris to semitendinosus.

\section{Case report}

An abnormal muscle bundle originated from long head of the biceps femoris was found in the right lower limb of adult male cadaver during gross anatomy dissection course at the Department of Anatomy in Ege University, Faculty of Medicine. The origin of the long head of biceps femoris was normal and most of the muscle fibers formed to tendon and was inserted on to the head of the fibula. But some of the proximal fibers from medial side at the mid-thigh level formed a separate muscle bundle and continued medially between biceps femoris and semitendinosus muscles as a narrow muscular slip. The muscle bundle then joined the distal portion of the semitendinosus muscle as it traveled obliquely and inferiorly. The muscular bundle runs across the sciatic nerve along its course. It crossed closely to the sciatic nerve along its course. The total length of the muscle bundle from the most proximal point to the most distal point was $86.32 \mathrm{~mm}$ and the average width was $5.47 \mathrm{~mm}$. There were no other musculoskeletal anomalies found in the lower extremity and there were no relevant medical history findings for the cadaver (Figure 1). 


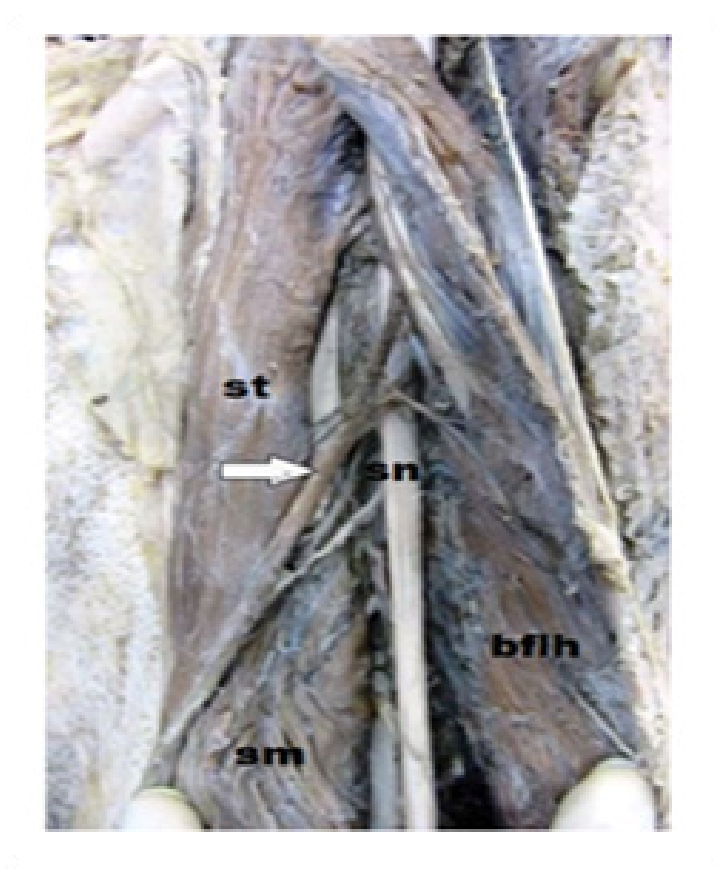

Figure I Muscle bundle indicated by white arrow. An abnormal muscle bundle originated from long head of the biceps femoris and is running to the semitendinosus muscle.

St, semitendinosus muscle; SM, semimembranosus muscle; BFLH, longhead of biceps femoris muscle; $\mathrm{SN}$, sciatic nerve

\section{Discussion}

The anatomical organization and architecture of hamstring muscle complex have been studied for many years with the aim to provide more detailed anatomical information about hamstring muscles. A review of the literature showed different variations in the hamstring muscle complex. The variations of hamstring muscle complex can be classified as fallow: an accessory semimembranosus muscle, ${ }^{6}$ hypoblastic or absent semimembranosus, ${ }^{7}$ duplicated semimembranosus muscle, ${ }^{8}$ common proximal tendon of three muscle, ${ }^{9}$ separate distal short head of biceps brachii insertion, and separate proximal long head of biceps brachii. ${ }^{2,10}$ However, some of the muscular variations such as a muscular slip, which originated from hamstring muscles are only found in extremely rare cases. The aberrant muscular bundle found in this case arose from the long head of biceps femoris and merged with the semitendinosus. There is very little literature to reports existence of an anomalous muscle or muscular bundle between the long head of biceps femoris and semitendinosus muscles.

The presence of the aberrant muscular bundle between the long head of biceps femoris and semitendinosus muscles was described for the first time by Sinav et al. ${ }^{11}$ The aberrant muscular bundle in their report described an origin from $12 \mathrm{~cm}$ below the ischial tuberosity that joined the long head of biceps femoris at $12.5 \mathrm{~cm}$ above the medial epicondyle. More recently, Bang et al. ${ }^{12}$ reported a similar muscular bundle between the long head of the biceps femoris and semitendinosus muscles. The muscular bundle in their report originated from the long head of the biceps femoris, which was approximately $127.7 \mathrm{~mm}$ lower than was the ischial tuberosity and merged with the semitendinosus $110.5 \mathrm{~mm}$ above its insertion; its length was $138.8 \mathrm{~mm}$. Location and course of the muscular bundle in the present case is similar to that of the previous two cases. The innervations of the muscular bundle by the tibial component of the sciatic nerve are the same as other cases; however, the total length of muscular bundle in our case is relatively shorter than that of the other cases.

Such variations may change the biomechanics of the muscles. Such a muscular bundle which connects the long head of the biceps femoris to the semitendinosus is likely to increase the strength of semitendinosus and thus produce strong internal rotation in the semi flexed knee. Therefore the smaller muscular bundle in the present case may exhibit more of an effect on strain and function of the semitendinosus. Because of the close relationship with the sciatic nerve, this type of muscular slip may compress the nerve and cause sciatic nerve entrapment and a mysterious pain syndrome in the legs. Therefore, the existence of such a muscular variation in this cadaver could have cause unexplained nerve entrapment syndromes in the leg during his life. Clinically, such a rare muscular variations may cause confusion and may be mistaken for soft tissue tumors.

Another muscular anomaly between the long head of the biceps femoris and the semitendinosus has been identified by Chakravarthi. ${ }^{13}$ Chakravarthi reported an anomalous muscle, not a muscular bundle or slip, which originated from the long head of biceps femoris and merged with the semitendinosus. The length of this muscle was $6.5 \mathrm{~cm}$; and its breadth was $3.5 \mathrm{~cm}$. He has suggested that it may be a third head of biceps femoris due to its size and location. Therefore it is also possible that an accessory muscle long head of biceps femoris to semitendinosus. Being aware of variations in hamstring muscles is essential not only for anatomist but also for surgeons and radiologist and may prevent diagnostic errors.

During limb myogenesis in vertebrates, muscle precursors enter the limb bud and they separate to dorsal and ventral muscle mass neighbour to the cartilage-forming mass. After that, both domains are gradually split into three components along the proximal-distal axis to develop many identifiable muscles domains. Specific muscles of the limb are formed as a result of continuous migration of muscle precursor through the proximal limb at late myogenesis. ${ }^{14}$ Existence of an aberrant muscular bundle in the present study may have resulted from defect in the migration process of muscle precursor during to embryogenesis.

\section{Conclusion}

In conclusion, we found an aberrant muscular bundle which connects the long head of biceps femoris to semitendinosus muscle. The presence of such an anomalous bundle is of specific importance because of its potential to cause hamstring strain and sciatic nerve compression. This muscular bundle may also cause confusion during surgery or evaluation of CT and MRI scans. Therefore, being aware of this variation is necessary to avoid complications during radiodiagnostic procedures or surgeries.

\section{Acknowledgements}

None.

\section{Conflict of interest}

Author declares that there is no conflict of interest. 


\section{References}

1. Beltran L, Ghazikhanian V, Padron M, et al. The proximal hamstring muscle-tendon-bone unit: a review of the normal anatomy, biomechanics, and pathophysiology. Eur J Radiol. 2012;81(12):3772-3779.

2. Feucht MJ, Plath JE, Seppel G, et al. Gross anatomical and dimensional characteristics of the proximal hamstring origin. Knee Surg Sports Traumatol Arthrosc. 2015;23(9):2576-2582.

3. Linklater JM, Hamilton B, Carmichael J, et al. Hamstring injuries: anatomy, imaging, and intervention. Semin Musculoskelet Radiol. 2010;14(2):131-161.

4. Opar DA, Williams MD, Shield AJ. Hamstring strain injuries: factors that lead to injury and re-injury. Sports Med. 2012;42(3):209-226.

5. Koulouris G, Connell D. Hamstring muscle complex: an imaging review. Radiographics. 2005;25(3):571-586.

6. Stoane JM, Gordon DH. MRI of an accessory semimembranosus muscle. J Comput Assist Tomogr. 1995;19(1):161-162.

7. Peterson JE, Currarino G. Unilateral absence of thigh muscles confirmed by CT scan. Pediatr Radiol. 1981;11(3):157-159.
8. Cattrysse E, Barbaix E, Janssens V, et al. Observation of a supernumerary hamstring muscle: a state of the art on its incidence and clinical relevance. Morphologie. 2002;86(274):17-21.

9. Markee JE, Logue JT Jr, Williams M, et al. Two-joint muscles of the thigh. J Bone Joint Surg Am. 1955;37(1):125-142.

10. Sato K, Nimura A, Yamaguchi K, et al. Anatomical study of the proximal origin of hamstring muscles. J Orthop Sci. 2012;17(5):614-618.

11. Sinav A, Gümüşalan Y, Arifoğlu Y, et al. Accessory muscular bundles arising from biceps femoris muscle. Kaibogaku Zasshi. 1995;70(3):245-247.

12. Bang JH, Gil YC, Yang HJ, et al. Multiple muscular variations in the neck, upper extremity, and lower extremity biased toward the left side of a single cadaver. J Korean Med Sci. 2015;30(4):502-505.

13. Chakravarthi K.Unusual unilateral multiple muscular variations of back of thigh. Ann Med Health Sci Res. 2013;3(Suppl 1):S1-S2.

14. Schnorrer F, Dickson BJ. Muscle building; mechanisms of myotube guidance and attachment site selection. Dev Cell. 2004;7(1):9-20. 